Arqueología y Sociedad,

$N^{o} 18,2007$

\title{
Panorama de los santuarios inca de alta montaña en Argentina
}

\author{
María Constanza Ceruti*
}

\begin{abstract}
Resumen
La ocupación de los Andes meridionales en época Inca incluyó la apropiación física de espacios de cumbres de más de 5.000 metros sobre el nivel del mar para la realización de sacrificios y ofrendas articulados bajo las ceremonias de capacocha. La prospección de varias decenas de cimas en distintos sistemas orográficos del territorio argentino, desde los volcanes que jalonan la altiplanicie puneña hasta los picos cordilleranos que señalan los confines del imperio Inca, representa un amplio substrato de evidencia arqueológica susceptible de ser interpretado a la luz de las fuentes históricas, con el auxilio de modelos de base etnográfica y etnoarqueológica. En este trabajo se pone de manifiesto parte de la variabilidad de los santuarios de alta montaña documentados por la autora en Argentina, dando cuenta de su diversidad en relación a los usos sociales, el propósito político y el simbolismo religioso implicados en la activa sacralización de la geografía andina como parte de las estrategias de legitimación de la dominación imperial Inca.
\end{abstract}

Palabras clave

Santuarios, cumbres, inca, sacrificios, ofrendas, Argentina.

\begin{abstract}
The Inca occupation of the Southern Andes involved the physical access to summits over 5.000 meters above sea level for the purpose of sacrifices and offerings articulated within the frame of the capacocha ceremonies The survey of dozens of peaks in diverse orographic systems in the argentine territory from the volcanoes in the Puna highlands to the mountain ranges in the southernmost corner of the Inca empire conform a wide range of archaeological data which can be interpreted with the help of historical sources, as well as ethnographic and ethno-archaeological models. In this paper we approach the diversity of the mountaintop shrines surveyed and excavated by the author in Argentina, which is interpreted in relation to the social use, political purpose and symbolic meaning of the sacred geography in the strategies of legitimization of the Inca domination in the Andes.
\end{abstract}

\section{Keywords}

Shrines, summits, inca, sacrifices, offerings, Argentina.

\footnotetext{
* Consejo Nacional de Investigaciones Científicas (CONICET). Universidad Católica de Salta .Correo electrónico: constanza_ceruti@yahoo.com
} 


\section{Introduccion}

Decenas de santuarios de altura fueron erigidos hace aproximadamente medio milenio por la civilización Inca en cumbres de Perú, Bolivia, Chile y Argentina. Bajo la dominación del imperio cuzqueño se logró, por primera vez en la historia de la humanidad, el ascenso exitoso a cimas de más de 6.000 metros de altitud para la realización de ofrendas y sacrificios humanos. Dichas ofrendas y sacrificios tenían lugar en el marco de ceremonias estatales conocidas como capacochas, las cuales se realizaban a la muerte del emperador Inca, en ocasión de catástrofes naturales (sequías, erupciones volcánicas) o bien para la propiciación de la fertilidad y la prosperidad.

Evidencias de carácter bioantropológico y artefactual de las capacochas incaicas en altura han sido descubiertas en montañas del sur de Perú en particular en los volcanes de la región de Arequipa y del Cañón de Colca. Tal es el caso de la momia juvenil femenina conocida como Juanita, y de los demás enterratorios excavados por el antropólogo norteamericano, Johan Reinhard y por el Prof. José Antonio Chávez en el nevado de Ampato; así como en el Pichu Pichu, en el monte Sara Saray en el volcán Misti ${ }^{1}$ (ver Reinhard 1996, 1997, 1998 y 2005) .

Un gran número de los sitios ceremoniales de alta montaña fueron construidos también en el territorio que corresponde actualmente al noroeste de la República Argentina, el cual formaba parte del Collasuyu, la provincia meridional del Incanato (Fig. 1). Las investigaciones arqueológicas de altura desarrolladas por la autora desde 1996, otorgan continuidad a los trabajos pioneros que, desde treinta años antes venían efectuando en las cumbres del oeste de Argentina el arqueólogo suizo-argentino, Juan Schobinger y el montañista italo - argentino Antonio Beorchia Nigris. Entre 1999 y 2000, la subscripta codirigió con Johan Reinhard, cuatro campañas de excavación arqueológica en el norte de Argentina, incluyendo el descubrimiento y recuperación de tres momias congeladas y aproximadamente cien ofrendas asociadas, procedentes del sitio ceremonial más elevado del planeta, en la cima del volcán Llullaillaco. Por otra parte, la autora ha dirigido proyectos de investigación en las provincias argentinas de San Juan, La Rioja y Jujuy.

Resultante de diez años de trabajo de campo en arqueología de alta montaña, la presente contribución procura ofrecer un panorama de la diversidad en los santuarios de altura documentados en el oeste y el noroeste de Argentina, ofreciendo algunas interpretaciones tentativas en torno a los propósitos políticos y el simbolismo religioso implicados en su construcción y utilización por parte del Estado Inca.

\section{Las máxima alturas de Mendoza y San Juan: sacralizando los confines del imperio}

El Aconcagua, situado en el confín meridional del territorio del imperio Inca, fue considerado una montaña sagrada y homenajeado mediante una ceremonia de capacocha efectuada en su ladera occidental. Así lo sugiere el rescate de un niño momificado, enterrado con ajuar mortuorio a 5.300 metros de altura en la base del contrafuerte conocido como Pirámide intervención que fuera efectuada por Juan Schobinger en 1985 (ver Schobinger 2001). Entre las motivaciones que habrían dado cuenta de la ceremonia se ha sugerido la sacralización de la frontera meridional del imperio, así como la consolidación de una alianza con autoridades araucanas en la región (Gentile 1996).

\footnotetext{
1 En 1998, la que suscribe fue invitada por el Dr. Reinhard a participar de las investigaciones arqueológicas en el Misti, que se desarrollaron durante casi un mes, a más de 5800 metros de altitud, en el interior del cráter activo del volcán, resultando en el hallazgo y recuperación de individuos acompañados de ajuar, en pobre estado de preservación como consecuencia de las emanaciones sulfurosas del domo.
} 


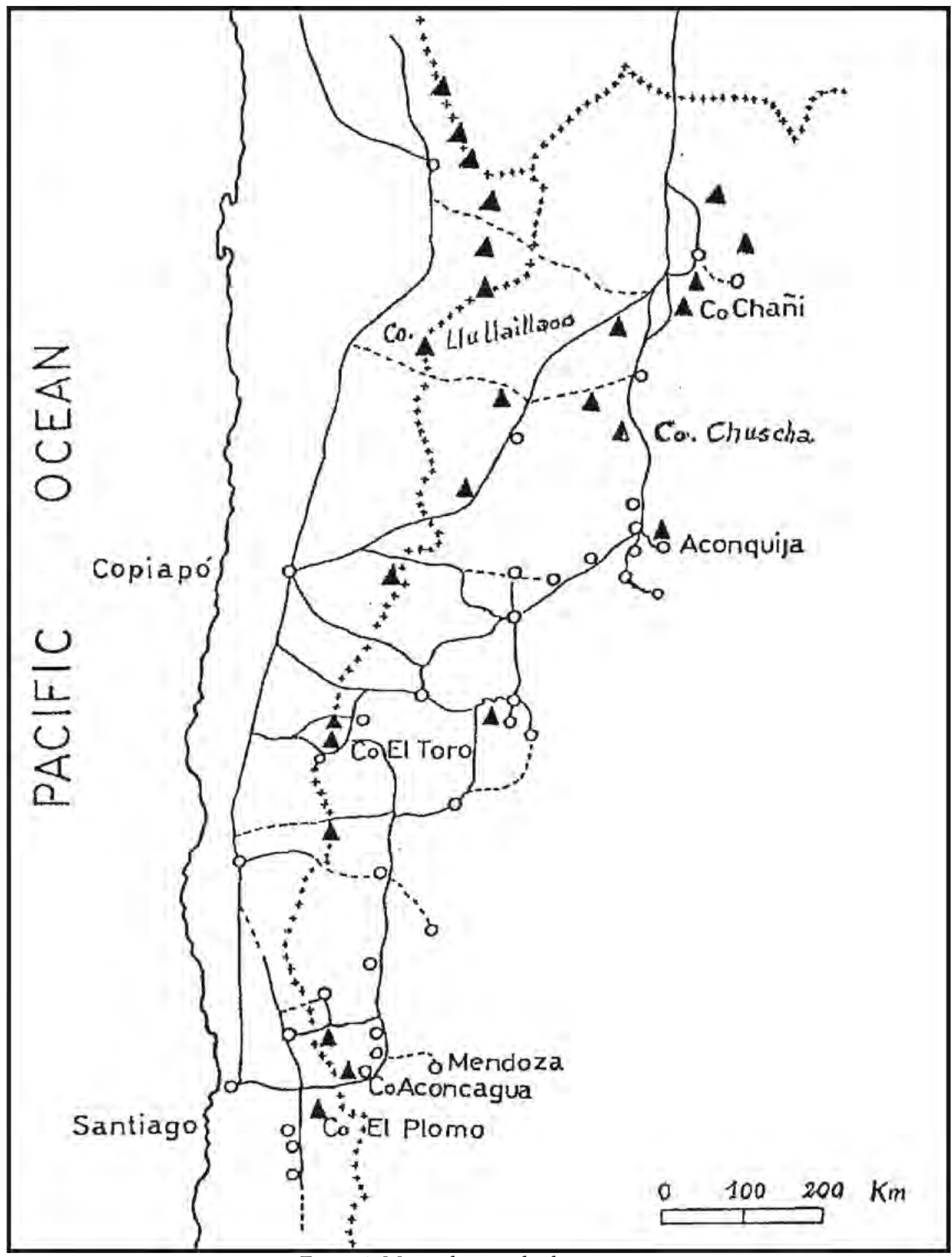

Figura 1. Mapas de sitios de alta montaña. 
Cabe señalar que móviles semejantes, de índole sociopolítica, aparecen explícitamente vinculados al uso de los escenarios de montaña en ceremonias sacrificiales de capacocha, según lo referido en las fuentes históricas. Tal es el caso de Tanta Carhua, hija del cacique de Ocros, quien de acuerdo a lo registrado por el cronista Rodrigo Hernández Príncipe (1986 [1621]), había sido sacrificada y enterrada en un monte caracterizado como mojón fronterizo de la expansión cuzqueña, a cambio de la reconfirmación de su padre en el cargo de curaca.

El uso que los Incas hicieron de la escenografía sacralizada del monte Aconcagua parece haber sido breve. Dicha montaña no llegó a convertirse en un centro de peregrinaje masivo, como sí lo fueron algunas de las montañas situadas más al Norte. Es de suponer que la ceremonia en el contrafuerte Pirámide habría sido ejecutada por un grupo reducido de oficiantes y que habría tenido lugar en una topografía poco conocida para ellos: el niño fue enterrado a los pies de un paso rocoso de alta dificultad, que presumiblemente ofreció a los sacerdotes incas un obstáculo insuperable, al no contar con medios técnicos de escalada. Por otra parte, la ausencia de evidencias arqueológicas sobre la ruta de más fácil acceso en el Aconcagua, contribuye a suponer que no se llegó a explorar dicha vertiente en épocas prehispánicas, por lo que tampoco parece probable que los Incas hayan alcanzado la cima (Ceruti 1999a).

En las inmediaciones del monte Aconcagua, la autora visitó un santuario incaico de segundo orden construido en la cumbre del cerro Penitentes (4.352 m), el cual fue estudiado por el arqueólogo argentino Roberto Bárcena (Schobinger 2001). Por otra parte, sobre la vertiente occidental de los Andes, en las inmediaciones de la ciudad de Santiago de Chile, se encuentra el santuario de altura del nevado del Plomo. En la década del cincuenta, buscadores de tesoros que habían ascendido la montaña extrajeron el cuer- po momificado naturalmente por congelamiento de un niño de unos ocho años de edad, dotado también de vestimenta y atributos compatibles con los de una ceremonia de capacocha Inca (Mostny 1957).

Dominando la vista desde la cima del Aconcagua hacia el Norte se yergue el imponente perfil del Mercedario, montaña de $6.770 \mathrm{~m}$ de altitud cubierta de extensas corazas glaciarias. Desde su cumbre se avista el océano Pacífico, a la vez que sus glaciares dan vida a importantes ríos, todos ellos criterios orográficos que las fuentes históricas - en particular la "Instrucción para descubrir todas las guacas del Piru y sus camayos y haziendas" de Cristóbal de Albornoz [1583] (Duviols 1967) - señalan como directamente relacionados con la percepción de la sacralidad de las montañas andinas en épocas prehispánicas.

Las laderas del Mercedario están dotadas de un importante complejo ceremonial de alta montaña de época Inca. El ascenso a la cima efectuado por la autora constituyó la primera campaña científica a las máximas alturas del coloso, permitiendo el descubrimiento de estructuras ceremoniales en la cota de los $6.400 \mathrm{~m}$ y haciendo posible completar las tareas de exploración y relevamiento iniciadas por Juan Schobinger en la base de la montaña (Schobinger 1968) y continuadas a mayor altura por Antonio Beorchia, a comienzos de los años setenta (Beorchia 1985). La distribución de la arquitectura ceremonial y logística, así como la presencia de un santuario satélite en las alturas del vecino nevado Alma Negra $(6120 \mathrm{~m})$, permiten considerar al Mercedario entre los complejos ceremoniales de montaña más importantes de los confines meridionales del imperio incaico (Ceruti 2003e).

En la cordillera al norte de la provincia de San Juan se yergue el cerro El Toro $(6.160 \mathrm{~m})$, que fuera escenario del hallazgo de una momia masculina adulta, descubierta por Erico Groch y Antonio Beorchia, y rescatada por Juan Schobinger en 1964 (Schobinger 1966b). En la 
prospección efectuada por la autora se recorrió la vertiente meridional, que hasta entonces permanecía inexplorada, sin encontrarse rastros de utilización prehispánica. La escalada que la subscripta efectuara a las vecinas cimas de los cerros Coipita $(5.423 \mathrm{~m}$ ) y Amarillos (5.600 m), al igual que una posterior ascensión a la cima del cerro El Potro $(5.730 \mathrm{~m})$, tampoco revelaron datos de interés arqueológico.

Tanto el cerro el Toro como el monte Mercedario se encuentran asociados con importantes instalaciones logísticas de época Inca ubicadas cerca de sus bases y conocidas en el área como "tamberías". Tal es el caso de la tambería de Pircas de Indios, sobre las laderas bajas del Mercedario (Ceruti 2003e; Schobinger 1968), y de la tambería de Paso Valeriano, a los pies del cerro el Toro (Schobinger 1966b). La palabra quechua tampu, hispanizada como "tambo", describe a las instalaciones de hospedaje y aprovisionamiento dispuestas junto a los caminos incaicos (Hyslop 1990). Habitualmente, se ha contemplado la existencia y la ubicación de las tamberías junto a los pasos montañosos de las provincias de Mendoza y San Juan en relación a las necesidades logísticas de los cruces cordilleranos (Bárcena 1998); aunque también fue propuesta su vinculación, en el plano simbólico, con los santuarios de altura en las inmediaciones (Schobinger 1966b).

Sin embargo, las tamberías cordilleranas adquieren otra dimensión si se considera su probable uso social como campamentos base para las ascensiones a los santuarios en las cimas vecinas. Dicho enfoque daría cuenta de la importante inversión en monumentalidad de la arquitectura ceremonial incorporada a algunos de los tambos incaicos en la base de ciertas montañas, tal como el Pueblo Viejo, a los pies del volcán Licancabur, en el norte de Chile (Barón y Reinhard 1980; Ceruti 2005c), o el mismo tambo de Paso Valeriano. Por otra parte, cabe suponer el uso que dichos campamentos base habrían tenido, como lugares para aclimatación de los sacerdotes y auxiliares destinados a ascender hasta las máximas alturas para el desarrollo de las ceremonias. Según lo sugieren las fuentes históricas, los tambos asociados a centros religiosos y por ende destinados a albergar peregrinos, recibían el nombre de corpahuasis (Ramos Gavilán 1976: 66).

\section{Los santuarios de altura en las montañas de La Rioja: entre lagunas sagradas y reservas mine- ras}

Al norte de San Juan, adentrándose en territorio de la provincia de La Rioja, la cordillera andina comienza a abrirse, ofreciendo un paisaje en el que se conjugan grandes nevados, cerros de mediana altura y extensos espejos de aguas, como el de la Laguna Brava. Tratándose de un espacio sumamente desértico y poco apto para el asentamiento humano, el ceremonialismo relacionado con las montañas y lagunas sagradas andinas parece haber tenido un papel fundamental en la utilización que el Incanato planificara para esta región.

El nevado Veladero $(6.436 \mathrm{~m})$ se yergue al noroeste de la Laguna Brava, ostentando un imponente santuario de altura con arquitectura de típico estilo Inca, el cual fuera documentado sucesivamente por Antonio Beorchia, Johan Reinhard y la autora de este trabajo (Beorchia 2001, Ceruti 2004c). Las exploraciones de la subscripta se extendieron a las montañas vecinas, comprendiendo al cerro Fandango $(5.612 \mathrm{~m})$, emplazado al oeste de la Laguna Brava; el cerro Morado $(5.230 \mathrm{~m})$, ubicado al Norte del espejo de agua y los cerros Pilar (5.030 m) (Fig. 2)y Azul $(5.092 \mathrm{~m})$, que se elevan junto a la margen oriental. Todas estas cumbres ofrecen evidencias de apropiación ritual prehispánica que sugieren su articulación, a modo de santuarios satélites, con el principal escenario ceremonial de la cima del Veladero (Ceruti 2004c). Por otra parte, sobre la margen noroeste de la Laguna Brava se encuen- 


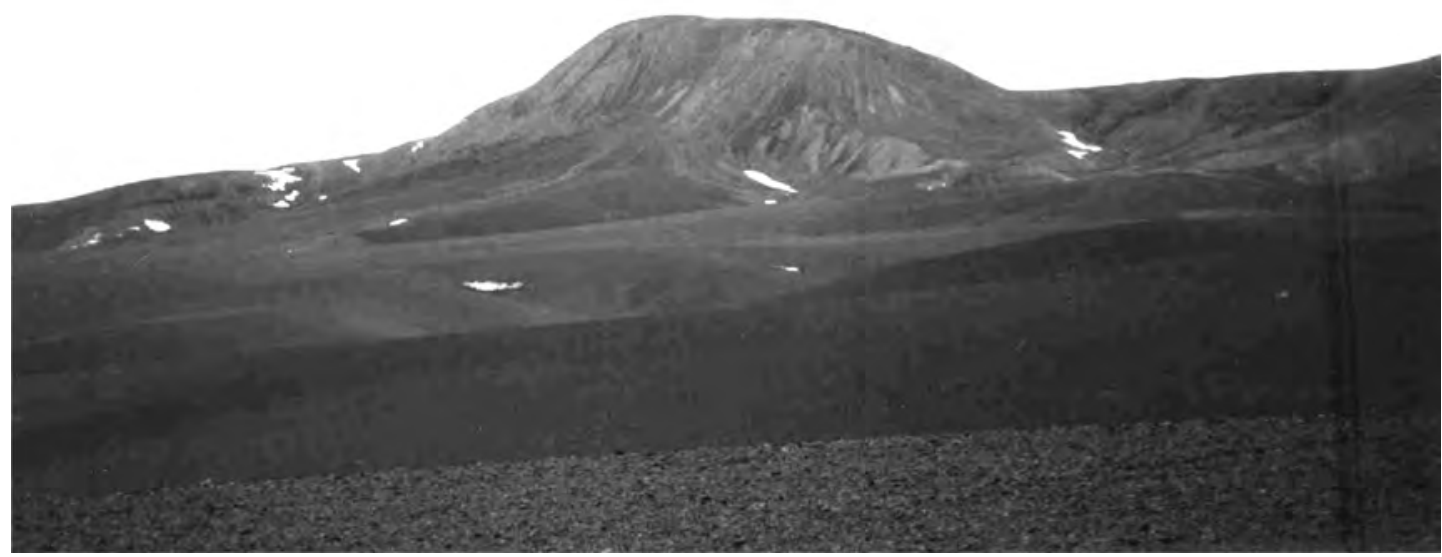

Figura 2. Vista del cerro Pilar.

tra emplazado un extenso tambo incaico, asociado a un inusual conjunto de plataformas ceremoniales ubicadas junto al borde mismo del espejo de agua. La asociación directa de las plataformas con la laguna sugiere que esta última también habría sido objeto de veneración (Ceruti 2003c)

El monte Pissis (6.882 m) y el cerro Negro de la Laguna Verde (5.764 m) se encuentran ubicados al norte de la cuenca de la Laguna Brava. El Pissis es el volcán más alto del mundo y la segunda cumbre de América, después del Aconcagua (Reinhard 1987). Es un macizo formado por cinco cumbres que superan todas ellas los 6.000 metros de altitud. En las observaciones realizadas por la autora sobre la extensa cumbre principal, no se lograron descubrir evidencias de ascensiones prehispánicas. Tampoco se encontraron vestigios arqueológicos en superficie en la cima del vecino cerro Negro de la Laguna Verde (Ceruti 2004d). Es posible que ambas montañas hubieran quedado aisladas de la red de santuarios de altura incaicos, por encontrarse ubicadas en un punto de la geografía andina no irrigado directamente por los caminos imperiales.

Los nevados de Famatina no forman parte de los Andes, puesto que pertenecen al sistema oro- gráfico de las Sierras Pampeanas, constituyendo una de las cadenas de serranías más altas del mundo. El macizo del Famatina se divide en dos grandes nevados que lo conforman: al Norte, el Negro Overo y al Sur, el General Belgrano. El santuario descubierto por la autora a 6.097 metros en la cumbre oriental General Belgrano del Famatina (Ceruti 2001a) presenta características muy similares al identificado inicialmente por Juan Schobinger (1966a) en el vecino cerro Negro Overo $(5.790 \mathrm{~m})$, montaña en la que la subscripta ha efectuado recientemente relevamiento de ruinas por encima de la cota de los $5.500 \mathrm{~m}$. Los sitios en las cumbres del Famatina se asemejan por presentar arquitectura de rectángulos ceremoniales, y astas de venado en carácter de ofrendas. Las astas de venado constituyen un rasgo regional circunscripto al Famatina y muy probablemente relacionado con la riqueza aurífera y la minería prehispánica (Ceruti 2004d). En este sentido, es necesario señalar que la relación entre la riqueza minera y los santuarios de altura ya había sido sugerida en el marco de las hipótesis explicativas en torno a la ocupación incaica del noroeste argentino (ver Raffino 1981) . 
Los nevados de los valles y la puna oriental de Salta: articulando centros ceremoniales y santuarios satélites

En el corazón del noroeste Argentino, los fértiles valles Calchaquíes fueron asiento de importantes desarrollos regionales durante el período previo a la dominación Inca en la región. La serranía del Cajón, que delimita el sector meridional del valle por el oeste, culmina en el nevado de Chuscha (5.512 m), que fuera escenario de una ceremonia prehispánica de capacocha. Las prospecciones efectuadas por la autora revelaron ausencia de restos arqueológicos en la cumbre principal del nevado de Chuscha, por resultar demasiado abrupta; aunque se identificaron evidencias de ocupación ritual prehispánica cumbres secundarias del nevado, que ofrecen superficies más llanas y extensas (Ceruti 2004e). Uno de esos emplazamientos debió ser el contexto original de entierro de una momia infantil femenina, perteneciente a una víctima de un sacrificio humano, que fuera sustraída por buscadores de tesoros durante los años veinte (Schobinger 2004). El folclore local la asocia con la presen- ciaincaica en la región, tal como se desprende de la leyenda de "la hija del rey Inga" (Ceruti 2004b) . Complementariamente, se documentó en el área un santuario de altura de menor jerarquía en la extensa cumbre del vecino cerro Bayo $(4380 \mathrm{~m})$. El sitio se encontraba alterado, con dispersión de tiestos cerámicos de estilo incaico en superficie. La cumbre del contiguo cerro Pabellón (5000 m), ostentaba una estructura de piedras apiladas cuyo carácter prehispánico no pudo ser establecido con certeza, en razón de la falta de elementos distintivos (Ceruti 2004e) . Otro tanto sucedió con la apacheta relevada a $4.600 \mathrm{~m}$ en la cercana abra o portezuelo de Pisca Cruz (Ceruti 2004f).

El nevado de Cachi se extiende hacia el norte por la margen occidental del valle Calchaquí. La autora tuvo oportunidad de visitar el importante santuario Inca a 6.150 metros, en la cima bautizada como "Melendez", a la vez que pudo observar depósitos de leña indicadores de ascensos prehispánicos en las cumbres menores denominadas Pirámide y Di Pasquo (Ceruti 1999a). Las prospecciones en los cerros

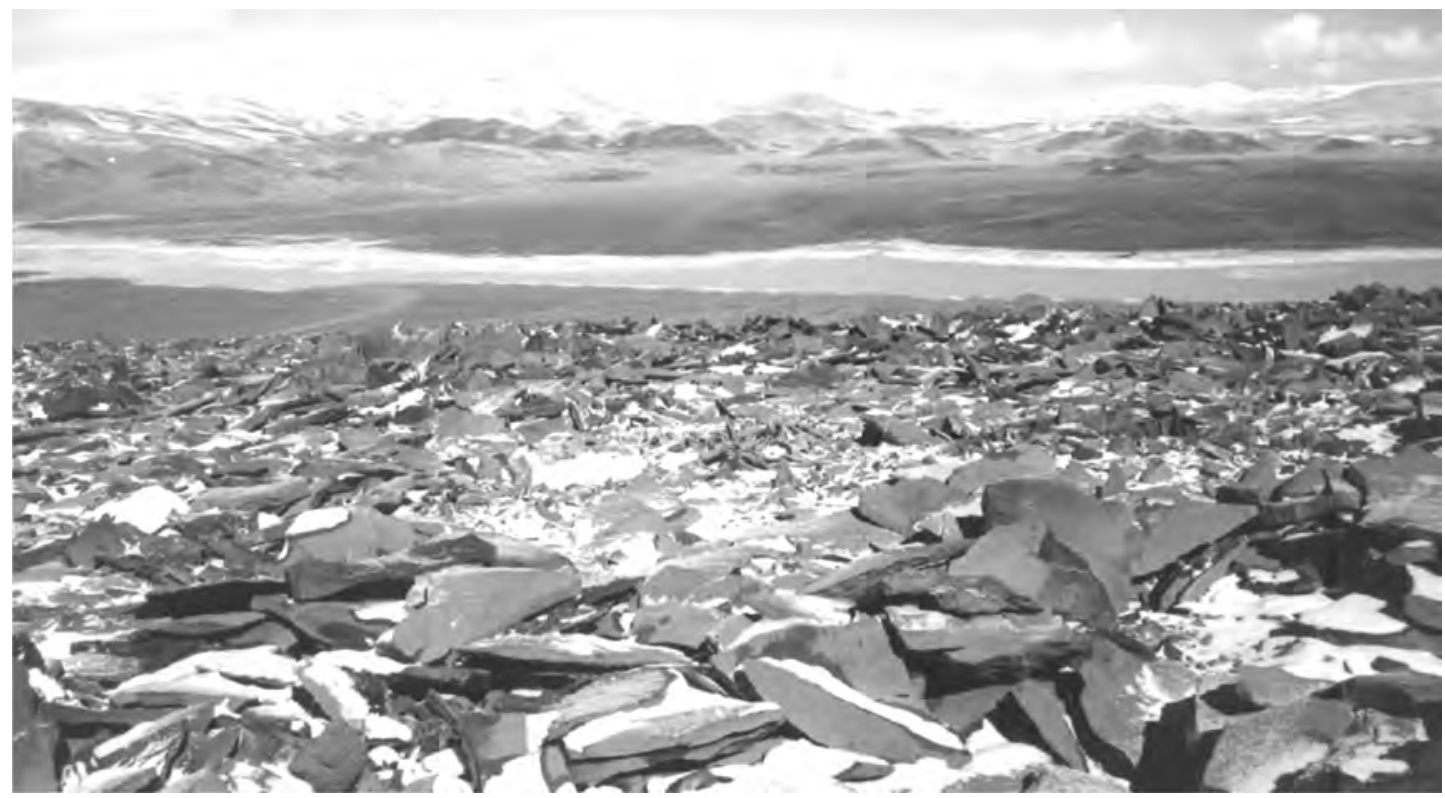

Figura 3. Vista de depósito de leña en el cerro Pilar. 
Malcante $(5.226 \mathrm{~m})$ y San Miguel de la Poma $(5.705 \mathrm{~m})$, que encierran al valle por el este, no arrojaron resultados arqueológicamente significativos (Ceruti 1999a).

En las nacientes de los valles Calchaquíes se levanta el nevado de Acay. Sucesivas ascensiones permitieron a la autora explorar la cima principal, de $5.716 \mathrm{~m}$ (Ceruti 2000b), y las cimas menores denominadas Blanca y Navaja (Ceruti 1999a). También se efectuó una campaña de excavación arqueológica en el santuario de la cumbre, en el marco de un proyecto de investigaciones dirigido en colaboración con el antropólogo Johan Reinhard. Lamentablemente, el avanzado estado de depredación del sitio sólo permitió documentar técnicas constructivas en el conjunto de plataformas incaicas, no siendo posible la recuperación de materiales artefactuales (Ceruti 2002 a e.p). Adicionalmente, la autora documentó sitios de menor jerarquía en los vecinos cerros Acay Chico (5.300 m.) y Saladillo (5.378 $\mathrm{m})$. Este último se encontraba saqueado por buscadores de tesoros mediante el uso de explosivos.

Dominando la puna oriental de Salta, al oeste del nevado de Acay, se yergue la mole del nevado de Quehuar $(6.130 \mathrm{~m})$. Durante un ascenso a la cumbre en 1996, la autora efectuó un reconocimiento de la arquitectura del santuario, que incluye un recinto circular de altos muros y una plataforma incaica o ushnu. La presencia de una rampa de acceso en el frente de la plataforma contribuye a la hipótesis de que la misma debió haber sido diseñada con una intencionalidad de uso escenográfico (Ceruti 1999b). En el marco de una expedición arqueológica codirigida con Johan Reinhard en Febrero de 1999, la cual constituyó la primera campaña de excavación sistemática en alta montaña en el Noroeste Argentino, se logró recuperar parte de un cuerpo humano congelado, el cual había sido parcialmente destruido por buscadores de tesoros (ver Beorchia 1985). Se recuperaron asimismo artefactos tales como una estatuilla femenina de valva de Spondylus, un fardo textil con ofrendas y escasos tiestos cerámicos, todos ellos dañados por la depredación y el saqueo que afectara intensamente al sitio. Pese a su fragmentario estado y precaria conservación, las evidencias son suficientes para afirmar que el enterratorio se habría efectuado en el marco de una ceremonia sacrificial de capacocha Inca (Ceruti 2001b; Reinhard y Ceruti 2005) .

En las montañas situadas en torno al nevado de Quehuar existen evidencias arqueológicas más modestas que también parecen haber sido originadas en el uso ritual que los Incas hicieron de cumbres de menor altura (aunque en la cota de los 5000 metros sobre el nivel del mar). Tal es el caso de las cimas de los volcanes Pocitos (5.020 m.) y Del Medio (4.930 m.), y de los cerros Quironcolo (5.202 m.), Aguas Calientes (5.397 m.) y Verde $(5.436$ m.). La prospección de sus cumbres permitió documentar arquitectura sencilla consistente en parapetos, rectángulos ceremoniales y recintos de muros bajos, con claros ejemplos de construcciones orientadas hacia la cima del Quehuar (Ceruti 2002b e.p.) . También se identificaron ofrendatorios prehispánicos sencillos en la cima del cerro Ratones (5.292 m) (Ceruti 2003f). En las publicaciones sobre el área referidas anteriormente, se ha propuesto un modelo de probable articulación, en la esfera del ritual, de los sitios en las montañas de menor altura con el santuario de mayor jerarquía situado en la máxima elevación. Los sitios de segundo orden habrían funcionado como "santuarios satélites" para el encendido de hogueras y la realización de actividades rituales complementarias al núcleo sacrificial de la ceremonia de capacocha, desarrollado en la cima de la montaña principal de la región.

\section{Los volcanes de la puna occidental de Salta: custodiando mensajeros a los dioses}

Al oeste del área de influencia del nevado de Quehuar se extiende la vasta puna occidental de 


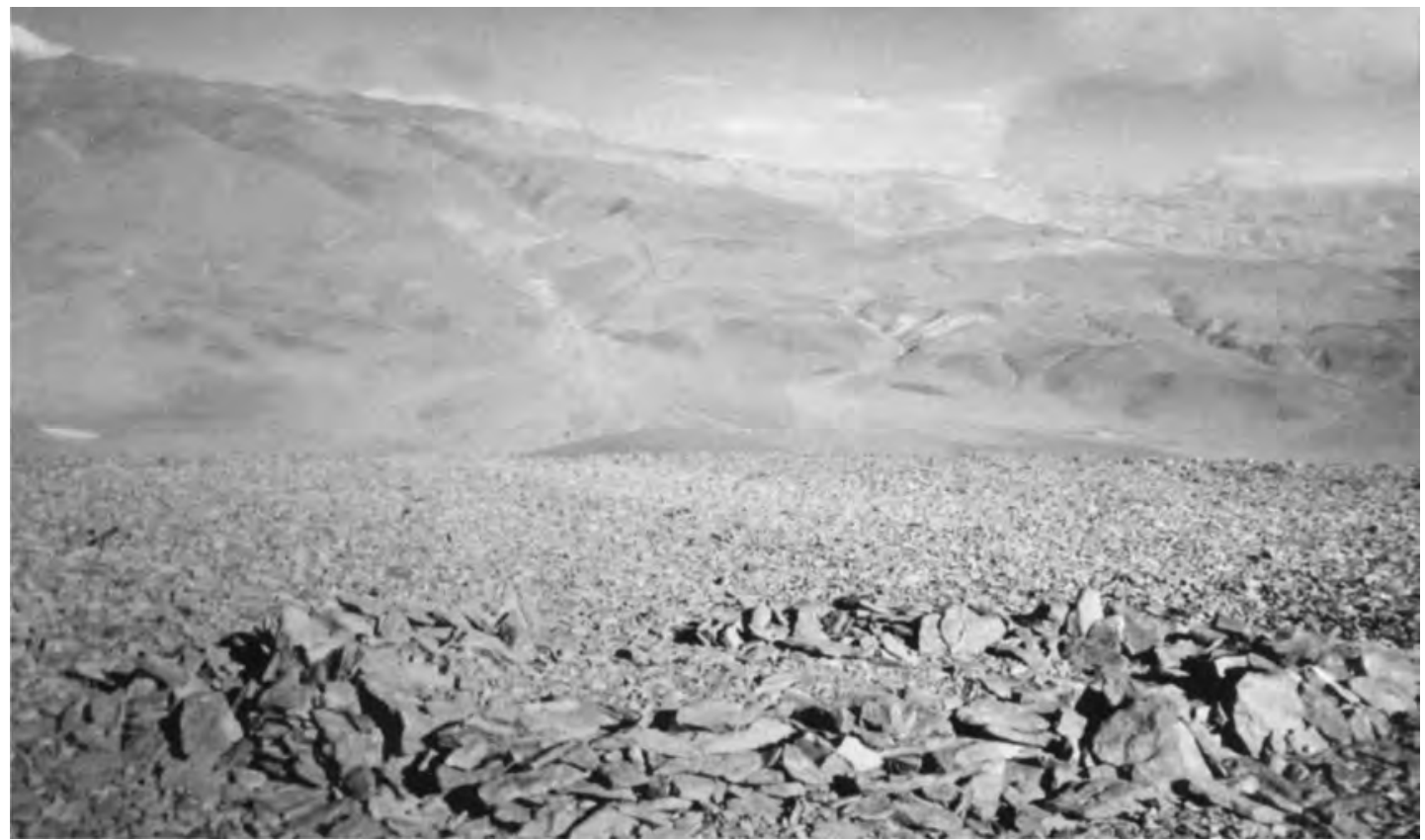

Figura 4. Rectángulo en la cuma de volcán Pocitos.

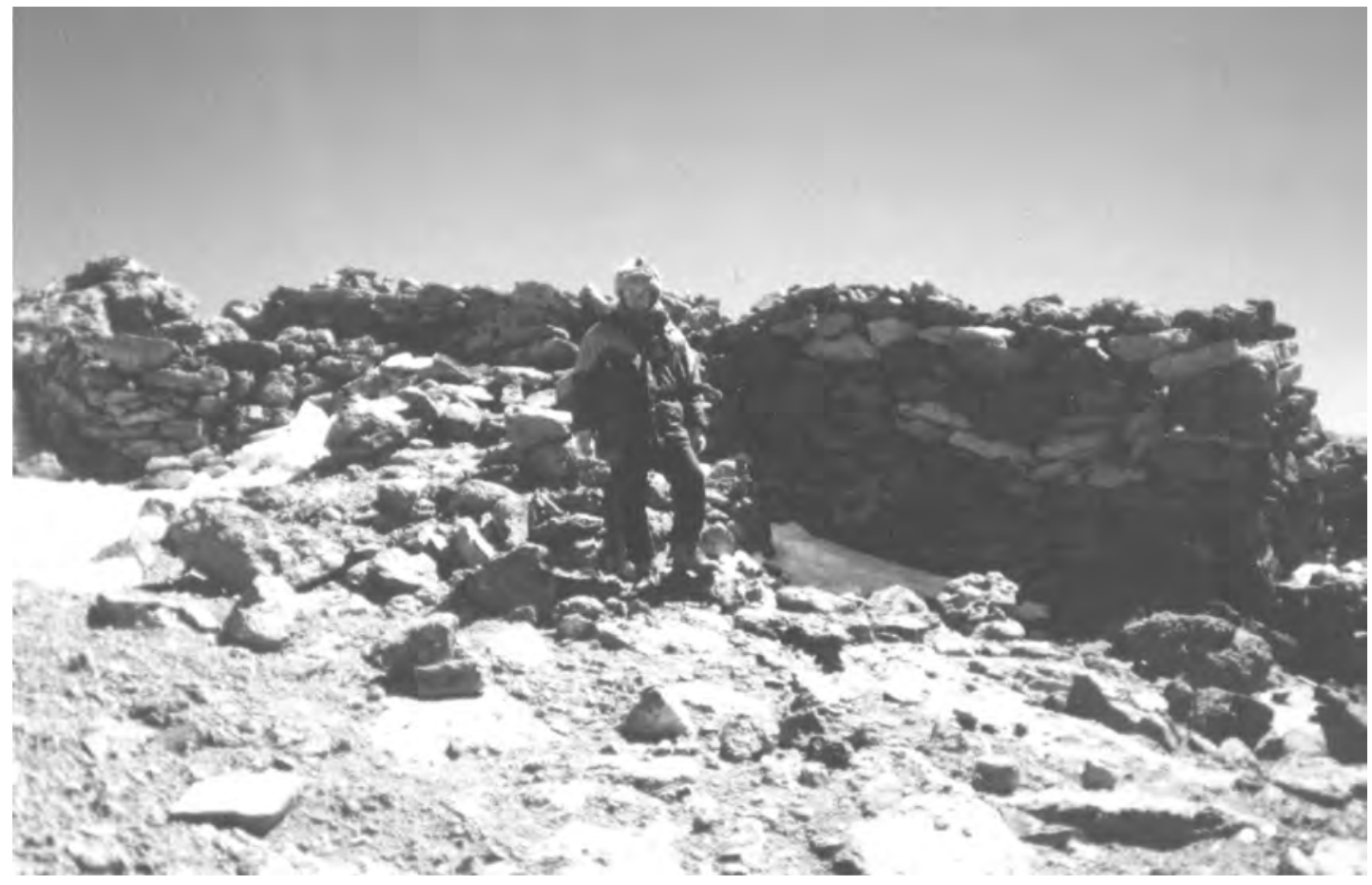

Figura 5. Plataforma ritual incaica en la cumbre del nevado del Quehuar. 
Salta, con volcanes que se encuentran rodeados por blancas salinas y extensos salares. En el seno de esta región, la ascensión de los cerros Rincón (5.594 m.) y Guanaquero (5.314 m.) condujo al descubrimiento y documentación de dos santuarios de altura de probable filiación incaica. Las ascensiones a los volcanes Arizaro (5.774 m.) y Blanco $(4.200 \mathrm{~m}$.) hicieron posible el releva- miento de dos sitios rituales con depósitos de leña; en tanto que la prospección sistemática de la cumbre del volcán Aracar (6095 m.) permitió documentar un santuario que pudo haber jugado un papel preponderante en la sacralización de la geografía del área. En una publicación específica (Ceruti 2001c), la subscripta ha caracterizado detalladamente a los sitios en cada una de estas cumbres, abordando con la ayuda de fuentes etnohistóricas la interpretación del uso simbólico y la función de los depósitos de leña allí encontrados, los cuales podrían haber servido como insumos para la quema de ofrendas, o bien como ofrendas en sí mismos (Anónimo 1968 [1590]:155; Molina 1959 [1575?]:79). La autora también publicó un santuario incaico con reutilización moderna documentado durante una ascensión a la cima de cerro Macón (Ceruti 1999c; 1998).

Las más altas montañas que trazan el límite entre la provincia de Salta (Argentina) y la región de Antofagasta (Chile) incluyen al volcán Socompa $(6.180 \mathrm{~m})$ y al volcán Llullaillaco (6.739 m.). Ascendiendo a la cima del Socompa por su vertiente sur - puesto que la ladera septentrional ya había sido explorada por Antonio Beorchia Nigris (1985) - se identificaron escasas evidencias de apropiación ritual del espacio en tiempos antiguos (Ceruti 1999a). Prácticamente estériles resultaron las exploraciones en las cumbres de los vecinos cerros Rosado (5.483), Mellado (5.317 m) y Socompa Caipis $(5.165 \mathrm{~m})$.

Indudablemente, la mayor contribución a las investigaciones arqueológicas en altura en Argentina tuvo lugar durante los trabajos en elvolcán Llullaillaco $(6.739 \mathrm{~m})$ codirigidos por la autora en colaboración con Johan Reinhard, quién ya había realizado prospecciones en esa montaña en la década de los ochenta (Reinhard 1993). Nuestras investigaciones arqueológicas sistemáticas en los diferentes sitios incaicos del volcán Llullaillaco se concretaron en Marzo de 1999, demandando 23 días de trabajo a más de 5.000 m.s.n. m., con una estadía ininterrumpida de 13 días en la cumbre, en una cota altitudinal cercana a 6.700 m.s.n.m. La expedición fue financiada con una beca otorgada a Johan Reinhard por la National Geographic Society. Contó con autorización de la Dirección de Patrimonio Cultural de Salta y con el apoyo del Ministerio de Educación, el Ejército, la Gendarmería y municipios de la región (Reinhard y Ceruti 2000) .

Durante los trabajos en el santuario a 6715 metros en la cumbre del Llullaillaco - considerado el sitio arqueológico más alto del mundo - se descubrieron y recuperaron tres cuerpos humanos pertenecientes a niños sacrificados durante una ceremonia de capacocha, que son considerados como las momias mejor preservadas de época precolombina conocidas hasta la fecha (Reinhard y Ceruti 2000, Ceruti 2003b y 2003g). Se encontraron asimismo alrededor de 100 objetos asociados a las momias, ya sea formando parte del ajuar colocado en las tumbas; o enterrados como ofrendas. Entre ellos, estatuillas de metal y valva de Spondylus ataviadas con miniaturas textiles y tocados de plumas; aríbalos y platos pato de cerámica; keros y cucharas de madera; chuspas tejidas conteniendo hojas de coca, charqui y maíz (Ceruti 2003a). Además se llevaron a cabo sondeos en el cementerio y en el tambo, dos sitios ubicados en la base del Llullaillaco, en altitudes que oscilan entre 4.900 y $5.200 \mathrm{~m}$.

El descubrimiento y puesta a resguardo de las momias del Llullaillaco y sus ajuares dio lugar a investigaciones interdisciplinarias coordinadas en el marco del Instituto de Investigaciones de 
Alta Montaña de la Universidad Católica de Salta, en colaboración con expertos internacionales y locales (Ceruti 2004a). Las investigaciones de laboratorio incluyeron estudios radiológicos (Previgliano et al 2003), estudios odontológicos (Arias Aráoz et al 2002), estudios de microbiología, anatomopatología y ADN antiguo (Reinhard y Ceruti 2000), análisis ceramológicos (Bray et al. 2005), estudios de cabello (Wilson et al. e.p.), estudios de textiles, entre otros.

En virtud de su extraordinaria conservación por congelamiento, los cuerpos de los niños momificados en la cima del volcán Llullaillaco permiten abordar aspectos relativos a la identidad y status social de las víctimas sacrificiales en el imperio Inca, contrastando mediante estudios bioantropológicos los requisitos de perfil sexoetario, belleza física y extracción social que las fuentes históricas refieren en relación a la selección de los mensajeros para el mundo de los dioses (ver Ceruti 2005b). Los niños del Llullaillaco no presentan deformaciones físicas ni afecciones dermatológicas altamente visibles que pudiesen atentar contra el ideal de belleza inmaculada reflejado en las crónicas (Ramos Gavilán 1976 [1621]: 62). Por otra parte, quedan perfectamente encuadrados dentro de los rangos de edad previstos para cada sexo - menos de diez años para los varones y hasta 15 años para las niñas (Murúa 1946 [1590]:342; Cobo 1990 [1653]:112) - ; a la vez que ofrecen un estado nutricional adecuado, compatible con una procedencia social elevada. Las diferencias en la deformación cefálica, apoyadas por los resultados de los estudios de A.D.N. mitocondrial, sugieren que las tres criaturas provenían de comunidades distintas (Reinhard y Ceruti 2000; Ceruti2005a).

\section{Los cerros de la puna de Jujuy: continuidades y cambios}

En el extremo norte de Argentina, las montañas de la Puna de Jujuy se diferencian de las res- tantes cumbres del noroeste del país por el hecho de que sus cimas no alcanzan altitudes tan extremas (no llegan en ningún caso a los 6000 metros) y porque se yerguen en medio de una topografía altiplánica más benigna y por ende más intensamente habitada por el hombre andino. La autora tuvo oportunidad de ascender a las cimas de los cerros Tuzgle $(5.486 \mathrm{~m})$, Collaguaima $(5.635 \mathrm{~m})$, Granada $(5.697 \mathrm{~m})$, Vilama $(5.678 \mathrm{~m})$, Crucesniyoq $(5.504 \mathrm{~m})$, Cuevas $(5.480 \mathrm{~m}) \mathrm{y}$ Zapaleri $(5.652 \mathrm{~m})$; entre otras montañas de la región (Ceruti 1999a y 2006). Según las observaciones de superficie, algunas de las cimas aparentan no haber sido ascendidas por los Incas, en tanto que otras ofrecen indicadores de utilización ritual prehispánica, a los que se suman las ascensiones subactuales, que ocasionalmente emprenden los mismos lugareños. En algunos casos, las cumbres presentan alteraciones resultantes de procesos tales como la erección de hitos geográficos y antenas repetidoras.

A semejanza de los valles Calchaquíes, la quebrada de Humahuaca, situada en el corazón de Jujuy, también fue asiento de importantes desarrollos culturales previos y contemporáneos a la conquista incaica. Dominando el fértil paisaje de Humahuaca se yergue el nevado de Chañi $(5.896 \mathrm{~m})$, dotado de un importante complejo ceremonial de altura y escenario de una capacocha Inca. Sus prominentes dientes de roca granítica separan la altiplanicie de las Salinas Grandes de las frondosas selvas nubosas de Jujuy, lo que determina la considerable variedad paisajística existente entre sus laderas oriental y occidental. En 1905, en el marco de una campaña militar, se sustrajo de las alturas del Chañi una momia infantil con ajuar textil, la cual quedó depositada en el Museo Etnográfico de Buenos Aires (Millán de Palavecino 1966; Ceruti 2001d).

En sucesivos ascensos al Chañi, la autora efectuó el relevamiento de la arquitectura del sitio de la cumbre y estaciones intermedias (Ceruti 1997a y 1997b). En Mayo del 2000 se lleva- 
ron a cabo excavaciones en el santuario de la cima, codirigidas con Johan Reinhard, orientadas a comprender en mayor profundidad el contexto en el que fuera realizada la ceremonia incaica que diera cuenta del enterratorio del infante mencionado con anterioridad. Las excavaciones se desarrollaron durante 15 días, con una estadía ininterrumpida de 10 días en la cumbre, en una cota altitudinal cercana a $6.000 \mathrm{~m}$ y con climatología invernal. Las condiciones del suelo para la excavación fueron extremadamente difíciles, debido a la presencia de nieve y al congelamiento del relleno de las estructuras, el cual en su contacto con el hielo había adquirido la consistencia del cemento. Debido a la depredación sufrida por el sitio, solo se recuperó evidencia fragmentaria, consistente principalmente en tiestos cerámicos y pedazos de hueso y madera (Ceruti 2002a e.p.).

\section{Discusión y conclusiones}

La exploración de cumbres que superan los 5000 metros de altura en territorio argentino ha contribuido a ampliar el panorama de la variabilidad de los sitios rituales de altura construidos en época Inca, permitiendo distinguir centros ceremoniales de primer orden y adoratorios de menor jerarquía. Estos últimos, en algunos casos, se habrían articulado con los primeros en carácter de santuarios satélites.

El modelo de los santuarios satélites parece estar presente en distintas regiones del oeste y norte argentino, desde la cordillera principal de San Juan hasta la puna de Salta. En las cumbres de menor jerarquía se reitera la presencia de depósitos de leña que indican actividades rituales complementarias, tales como el encendido de hogueras, o incluso el acarreo de cargas de leña para ser presentadas en sí mismas como ofrenda.

En la cordillera de La Rioja, la distribución de santuarios de alta montaña en el paisaje que circunda a la Laguna Brava sugiere la vinculación de los rituales incaicos en altura con aque- llos motivados por el carácter sagrado atribuido al espejo de agua. La presencia de ítems inusuales, como las astas de venado en las alturas del cordón de Famatina, sugieren articulación del ritual con actividades productivas de importancia regional, como la minería. En tanto que en algunas montañas de Jujuy, de menor altitud y de climatología más benigna, se encuentran vestigios de ascensiones andinas modernas que sugieren continuidad en el uso de los escenarios ceremoniales de altura.

Las cumbres más elevadas fueron escenario del entierro de ofrendas humanas y suntuarias en el marco de ceremonias incaicas de capacocha. Las capacochas involucraban la afluencia centrípeta - hacia la ciudad, el Cuzco - de niños y materias primas suntuarias procedentes de todo el imperio, como instancia previa a su redistribución, ya en carácter de ofrendas estatales, en procesión hacia los confines del Incanato (Duviols 1976). La existencia de grandes tambos en la base de algunas montañas sugiere su utilización como corpahuasis, para el albergue de los asistentes y peregrinos que hubiesen acompañado las procesiones.

El análisis de la evidencia bioarqueológica procedente de éstas montañas pone de manifiesto compatibilidad con la información suministrada por las fuentes históricas en relación al perfil de los principales actores sociales de los ritos de capacocha (Ceruti 2003a, 2004a; 2005a y 2005b). Los candidatos aceptables por su pureza para ser enviados a los dioses eran niños y mujeres vírgenes. Es así que el monte Aconcagua y el volcán Llullaillaco fueron lugar de entierro de varones de corta edad; en tanto que los nevados de Chuscha y Quehuar, al igual que el volcán Llullaillaco, custodiaron a niñas y a jóvenes doncellas. La creencia compartida, tanto por los conquistadores Incas como por las comunidades locales, era que después de la capacocha, los elegidos se convertían en emisarios en el mundo celeste, desempeñándose como guardianes y 
protectores de sus comunidades y del emperador (Gutiérrez de Santa Clara 1963 [1603]:233; Hernandez Príncipe 1986 [1621]: 473).

Los sacrificios y ofrendas efectuados en las altas montañas se justificaban ideológicamente en la conmemoración de eventos de la vida del soberano Inca, la expiación preventiva de culpas orientada a garantizar su bienestar y el de sus súbditos, la propiciación de la fertilidad de ganados y cosechas, el éxito en la guerra, así como el apaciguamiento de catástrofes naturales (Cobo 1990 [1653]; Polo de Ondegardo 1916 [1571]). Desde el punto de vista geopolítico, las procesiones en marcha hacia las principales montañas contribuían a sacralizar activamente la geografía andina y a consolidar la dominación Inca en los distantes territorios conquistados (Ceruti 2003a; Gentile 1996; Schobinger 2001).

Es interesante destacar que el fenómeno cultural de la apropiación de cumbres de más de 6000 metros con fines rituales es privativo del área andina y del período Inca. En los Himalayas, por ejemplo, las montañas sagradas son adoradas por circunambulación de la base pero no ascendidas hasta las cimas. Por su parte, la etnografía andina pone de manifiesto que las más altas cumbres de los Andes han dejado de ser visitadas con fines religiosos, puesto que el peregrinaje andino moderno se ha desplazado hacia cotas de altitud intermedias - alrededor de 4.700 metros sobre el nivel del mar - tal como ocurre en el caso de las procesiones al santuario de Punta Corral, en la provincia de Jujuy (Ceruti 1999a).

En razón de las dificultades en el acceso, los santuarios de altura no sucumbieron a los conquistadores españoles y extirpadores de idolatrías, conservando a través de los siglos, importantes evidencias materiales de los sacrificios y ofrendas efectuados por la civilización Inca. Es lamentable que en las últimas décadas, un alto porcentaje de los sitios arqueológicos en cumbres hayan sido depredados por buscadores de tesoros en eventos de huaqueo que, en muchos casos, terminaron en destrucción de la arquitectura; sustracción de las ofrendas y hasta dinamitación de restos humanos (Ceruti 1999a, 2003d). En otros casos, las alteraciones han sido el resultado no intencional de actividades topográficas, deportivas y mineras (Ceruti 2006) .

Desafortunadamente, las altas montañas continúan expuestas a la depredación; puesto que resulta virtualmente imposible designar custodios para que vigilen sitios a más de 5000 metros sobre el nivel del mar. Los yacimientos de altura presentan, en razón de su emplazamiento, la obvia limitación de no poder funcionar tampoco como "museos de sitio". En un contexto de inevitable exposición de las cimas como consecuencia del creciente desarrollo turístico y minero en áreas cordilleranas, las investigaciones arqueológicas en alta montaña constituyen una prioridad. Es de esperar que nuestras investigaciones contribuyan a suscitar conciencia acerca de la urgencia de preservar este inigualable legado del mundo Inca para las generaciones venideras.

\section{Agradecimientos}

Al Consejo Nacional de Investigaciones Científicas y Técnicas (CONICET) y a la Universidad Católica de Salta. A Juan Schobinger, Johan Reinhard y Antonio Beorchia Nigris. A los montañistas que me acompañaron en las diversas ascensiones y a las instituciones y personas que brindaron apoyo logístico para las campañas.

\section{Bibliografía}

Anónimo (Blas Valera)

1968 [1590] "Relación de las costumbres antiguas de los naturales del Pirú". En Crónicas peruanas de interés indígena, Biblioteca de autores españoles, editado por Francisco Esteve, tomo 209: 151-189. Madrid: Ediciones Atlas. 
Arias Araóz, Facundo, Josefina Gonzalez Diez y Constanza Ceruti

2002 "Estudios Odontológicos de las momias del Llullaillaco". Boletín de la Asociación Argentina de Odontología para Niños. Vol $31 \mathrm{~N}^{\circ}$ 2/3: 3-10. Buenos Aires.

Bárcena, Roberto

1998 Arqueología de Mendoza. Las dataciones absolutas y sus alcances. Mendoza: Editorial de la Universidad Nacional de Cuyo.

Beorchia Nigris, Antonio

1985 "El enigma de los santuarios indígenas de alta montaña". Revista del Centro de Investigaciones Arqueológicas de Alta Montaña, tomo 5. San Juan: Universidad Nacional de San Juan.

2001 Revista del Centro de Investigaciones Arqueológicas de Alta Montaña, tomo 6. La Plata: Universidad Católica de La Plata.

Bray, Tamara; Leah Minc; María Constanza Ceruti; José Antonio Chávez; Ruddy Perea y Johan Reinhard

2005 "A compositional analysis of pottery vessels associated with the Inca ritual of capacocha". Journal of Anthropological Archaeology 24:82100.

Ceruti, María Constanza

1997a "Sitios rituales de altura y estrategias sociales de dominación: caso de estudio en el nevado de Chañi (5.896 m., límite provinicial Jujuy-Salta, Argentina)". Revista de Estudios Sociales del N.O.A. Año 1 Nro 1: 127154. Tilcara: Instituto Interdisciplinario Tilcara (Facultad de Filosofía y Letras de la Universidad de Buenos Aires.

1997b La sagrada cumbre del Chañi: Los incas y el culto en las alturas vistos desde la
Arqueología. Tilcara: Instituto Interdisciplinario Tilcara (Facultad de Filosofía y Letras de la Universidad de Buenos Aires).

1998 Prospecciones en sitios de Alta Montaña en el Noroeste Andino Argentino: Informe Preliminar. Tawantinsuyu 5: 37-43. Canberra.

1999a Cumbres sagradas del nororeste argentino. Buenos Aires: Editorial de la Universidad de Buenos Aires (EUDEBA).

1999b "El complejo ceremonial de altura del nevado de Quehuar (6.130 m.s.n.m.; Depto. Los Andes; Pcia. de Salta): Un ejemplo de la dinámica incaica de interacción centro-periferia en el plano cúltico de la Capacocha”. En Actas del XII Congreso Nacional de Arqueología Argentina Tomo I: 186193. La Plata: UNLP.

1999c "El santuario de altura del cerro Macón (5.611 m.s.n.m.; Depto. Los Andes; Pcia. de Salta)”. En Actas del XII Congreso Nacional de Arqueología Argentina Tomo III: 292-296. La Plata: UNLP.

2000a "Santuarios de Altura del Nevado del Chuscha (5.512 m.s.n.m.) y del Cerro Bayo(4.612 m.s.n.m.) (Valles Calchaquíes, Pcia. de Salta, Argentina). En colaboración con Christian Vitry." Contribución Arqueológica $\mathrm{N}^{\circ}$ 5: 849-859. Copiapó: Museo Regional de Atacama.

2000b "El Santuario de Altura del Nevado de Acay (5.716 m.s.n.m.; Dpto. Los Andes; Pcia. de Salta, Argentina)." En colaboración con Christian Vitry. Contribución Arqueológica $\mathrm{N}^{\circ}$ 5: 841 848. Copiapó: Museo Regional de Atacama.

2001a "Santuario de altura en la cumbre 
Gral. Belgrano Este de los Nevados de Famatina (6.097 m.; Pcia. De La Rioja)". En Actas del XIII Congreso Nacional de Arqueología Argentina Tomo II: 367-374. Córdoba: Universidad Nacional de Córdoba.

2001b "Recientes hallazgos en los volcanes Quehuar (6.130 m.) y Llullaillaco (6.739 m.) ". En Actas del XIII Congreso Nacional de Arqueología Argentina. Tomo I:313-322. Córdoba: Universidad Nacional de Córdoba.

2001c "Aracar, Guanaquero, Rincón, Arizaro y Blanco: prospección y relevamiento de santuarios de altura en volcanes de la puna occidental salteña”. Relaciones de la Sociedad Argentina de Antropología Tomo XXVI: 145-166. Buenos Aires.

2001d "La Capacocha del Nevado de Chañi: Una Aproximación Preliminar desde la Arqueología”. Chungara 33 (2): 279-282. Arica: Universidad de Tarapacá.

2002 a (en prensa) "Excavaciones arqueológicas de alta montaña en el Nevado de Chañi (5.896 m.) y el Nevado de Acay $(5.716$ m.) (Provincia de Salta)". En Actas del XIV Congreso Nacional de Arqueología Argentina. Rosario: UNR.

2002b (en prensa) "Utilización ritual de cumbres en la puna oriental salteña: nuevos aportes para el estudio de los santuarios de altura de los Incas". En Actas del XIV Congreso Nacional de Arqueología Argentina. Rosario: UNR

2003a Llullaillaco: Sacrificios y Ofrendas en un Santuario Inca de Alta Montaña. Salta: Universidad Católica de Salta.

2003b "Archaeological find of three frozen- mummies and offerings at the Inca ceremonial complex on Mount Llullaillaco (Northwestern Argentina)". En Proceedings of the IV World Congress on Mummy Studies. Copenhagen: Danish Polar Center and Greenland National Archives in Nuuk.

2003c Santuarios de altura en la región de la Laguna Brava (Provincia de La Rioja, Noroeste Argentino): Informe de Prospección Preliminar. Chungara 35 (2): 233-252. Arica: Universidad de Tarapacá.

2003d Aspectos de la investigación, conservación y gestión del patrimonio cultural de alta montaña. En Conservación y Desarrollo de los ecosistemas de montaña, editado por el Ministerio de Relaciones Exteriores, Comercio Internacional y Culto, en base al Seminario realizado por el Año Internacional de la Montaña (Julio de 200). Buenos Aires: Editorial Dunken.

2003e "Mercedario y Alma Negra: Arqueología de Alta Montaña en el Departamento Calingasta (Provincia de San Juan)". Anales de Arqueología y Etnología Nº 54 / 55 (1999 / 2000): 47-80. Mendoza: Editorial de la Universidad Nacional de Cuyo.

2003f "Prospección arqueológica en el Cerro Ratones". Revista Andina N ${ }^{\circ} 37$. Cuzco: Centro de Estudios Andinos Bartolomé de las Casas.

$2003 \mathrm{~g}$ "First steps in the recovery and preservation of the frozen Inca mummies from volcano Llullaillaco (Northwestern Argentina)". En Proceedings of the IV World Congress on Mummy Studies. Copenhagen: Danish Polar Center and Greenland National 
Archives in Nuuk.

2004a "Human bodies as objects of dedication at Inca mountain shrines" (north-western Argentina). World Archaeology 36 (1):103-122.

2004b Toponimia y folklore en torno a las montañas sagradas del valle del Cajón. En el santuario incaico del Nevado de Chuscha. Compilado por Juan Schobinger. Mendoza: Editorial de la Universidad Nacional de Cuyo.

2004c "(en prensa). Prospección arqueológica del Nevado Veladero $(6.436 \mathrm{~m}$.) y de los cerros Azul (5.095 m) y fandango $(5.612 \mathrm{~m}$.) en la cordillera de La Rioja”. En Actas del XV Congreso Nacional de Arqueología Argentina. Río Cuarto: Universidad Nacional de Río Cuarto.

2004d Arqueología de Alta Montaña en La Rioja. Salta: Universidad Católica de Salta.

2004e "Revisión de los resultados de las prospecciones arqueológicas de alta montaña en el cerro Pabellón, el Nevado de Chuscha y el cerro Bayo". En El santuario incaico del Nevado de Chuscha, compilado por Juan Schobinger. Mendoza: Editorial de la Universidad Nacional de Cuyo.

2004f "Informe de Prospección Arqueológica al Abra de Pisca Cruz (4.640 m.) ". En El santuario incaico del Nevado de Chuscha. compilado por Juan Schobinger. Mendoza: Editorial de la Universidad Nacional de Cuyo.

2005a "Elegidos de los Dioses: Identidad y Status en las víctimas sacrificiales del volcán Llullaillaco y de otros santuarios de altura Inca". Boletín de Arqueología. 7 (2003): 263-275. Lima: Pontificia Universidad Católica del Perú. 2005b "Actores, ritos y destinatarios de las ceremonias incaicas de capacocha: una visión desde la arqueología y la etnohistoria". Xama 15-18 (2002. 2005): 287-299. Mendoza: CRICYT.

2005c "A la sombra del volcán Licancabur: santuarios de altura en los cerros Toco, Juriques y Laguna Verde". Xama 15-18 (2002-2005): 301-313. Mendoza: CRICYT

2006 Patrimonio Arqueológico de Alta Montaña en la Puna de Jujuy: algunas consideraciones. En Actas de las IV Jornadas en Honor a Guillermo Magrassi: Conocimiento Científico y Comunidad, de la Puna al Atlántico (Publicación electrónica). Centro de Estudios Indígenas y Coloniales de Jujuy y Museo Arqueológico Guillermo Magrassi.

Cobo, Fray Bernabé

1990[1653] Inca Religion and Customs. Editado por Ronald Hamilton. Austin: University of Texas Press. Duviols, Pierre

1967 Un inédito de Cristobal de Albornoz [1583?]: La Instrucción para descubrir todas las Guacas del Piru y sus camayos y haciendas. Journal de la Societe de Americanistes 56 (1): 7-39.

1976 "La Capacocha. Mecanismo y función del sacrificio humano, su proyección geométrica, su papel en la política integracionista y en la economía redistributiva del Tawantisuyu". Allpanchis: 9: 11-57. Cuzco.

Gentile, Margarita

1996 "La Dimensión sociopolítica y religiosa de la Capacocha del Cerro Aconcagua". Bulletin de L' Institut Francais d' Etudes Andines 25 (1): 43 90. Lima, IFEA. 
Gutiérrez de Santa Clara, Pedro

1963 [ca. 1603] Quinquenarios o Historia de las Guerras Civiles del Perú. En Biblioteca de Autores Españoles, tomo 166. Madrid: Ediciones Atlas.

Hernandez Príncipe, Rodrigo

1986[1621] "Idolatría del Pueblo de Ocros, cabeza desta comunidad". En Cultura Andina y Represión. Editado por Pierre Duviols, pp. 442-448. Cuzco: Centro de Estudios Rurales Andinos Bartolomé de las Casas.

Hyslop, John

1990 Inka Settlement Planning. Austin: University of Texas Press.

Millán de Palavecino, Delia

1966 "Descripción de material arqueológico proveniente de yacimientos de Alta Montaña en el área de la Puna”. Anales de Arqueología y Etnología 21: 81-99. Mendoza: Universidad Nacional de Cuyo.

Molina, Cristóbal de

1959 [1575?] Ritos y fábulas de los incas. Buenos Aires: Editorial Futuro. Murúa, Fray Martín de

1946 [1590] Historia del orígen y geneaología real de los reyes incas del Perú. Editado por Constantino Bayle. Biblioteca Missionalia Hispanica, vol. 2. Madrid.

Mostny, Grete

1957 "La momia del cerro el Plomo". Boletindel Museo Nacional de HistoriaNatural, tomo XXVII. Santiago: Museo de Historia Natural. Previgliano, Carlos, Constanza Ceruti, Johan Reinhard, Facundo Arias Araoz y Josefina González Diez

2003 Radiologic evaluation of the Llullaillaco Mummies. AJR 181: 1473-1479
Polo de Ondegardo, Juan

1916[1571] Informaciones acerca de la religión y gobierno de los incas. En Colección de libros y documentos referentes a la Historia del Perú, editado por Horacio Urteaga. Tomo III: 1-203. Lima: Sanmartí y Cía.

Raffino, Rodolfo

1981 Los Incas del Kollasuyu. La Plata: Editorial Ramos Americana.

Ramos Gavilán, Alonso

1976[1621] Historia de nuestra señora de Copacabana. La Paz: Editorial Universo.

Reinhard, Johan

1993 Llullaillaco: An investigation of the World's Highest Archaeological Site. Latin American Indian Literatures Journal pp. 31-54.

1987 “The Hidden Crater: Expedition to an unexplored plateau of Western Argentina". Optima 35 (4): 218-227

1996 "Peru's Ice Maidens". En National Geographic Magazine. 189 (6): 62-81. Washington D.C.

1997 "Sharp Eyes of Science Probe the Mummies of Peru". En National Geographic Magazine 191 (1): 36-43

1998 "Research Update: New Inca Mummies. "En National Geographic Magazine 194 (1): 128-135.

2005 The Ice Maiden: Inca Mummies, Mountain Gods and Sacred Sites in the Andes. National Geographic Society. Washington D.C.

Reinhard, Johan y Constanza Ceruti

2000 Investigaciones arqueológicas en el volcán Llullaillaco. Salta: Editorial de la Universidad Católica de Salta (EUCASA). 
2005 "Rescue Archaeology of the Inca Mummy on Mount Quehuar, Argentina". Journal of Biological Research LXXX (1): 303-307.

Schobinger, Juan

1966a "Investigaciones Arqueológicas en la Sierra de Famatina, provincia de La Rioja”. Anales de Arqueología y Etnología 21: 139-196. Mendoza: Universidad de Cuyo.

1966b "La momia del cerro El Toro: Investigaciones arqueológicas en la Cordillera de la Provincia de San Juan." Anales de Arqueología y
Etnología. Suplemento al tomo 21. Mendoza: Universidad de Cuyo.

2001 El santuario incaico del Cerro Aconcagua. Mendoza: Universidad de Cuyo.

2004 El santuario incaico del nevado de Chuscha. Mendoza: Universidad de Cuyo.

Wilson, Andrew, Constanza Ceruti, Thomas P. Gilbert, Timothy Taylor, Ian Barnes, Michael Worobey, Johan Reinhard, Michael P. Richards

2006 Diachronic aspects of human sacrifice the Llullaillaco Maiden. Publicación en preparación. Bradford: University of Bradford. 